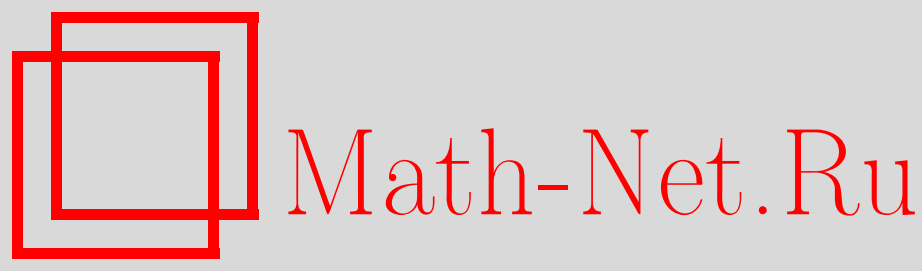

У. А. Розиков, Р. М. Хакимов, Ф. Х. Хайдаров, Крайность трансляционно-инвариантных мер Гиббса для модели Поттса на дереве Кэли, ТМФ, 2018, том 196, номер 1, 117-134

DOI: https://doi.org/10.4213/tmf9448

Использование Общероссийского математического портала Math-Net.Ru подразумевает, что вы прочитали и согласны с пользовательским соглашением http: //www.mathnet.ru/rus/agreement

Параметры загрузки:

IP : 54.198 .67 .100

26 апреля 2023 г., 06:23:27

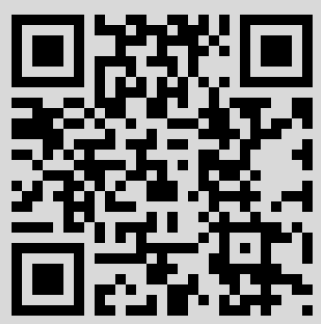




\title{
КРАЙНОСТЬ ТРАНСЛЯЦИОННО-ИНВАРИАНТНЫХ МЕР ГИББСА ДЛЯ МОДЕЛИ ПОТТСА НА ДЕРЕВЕ КЭЛИ
}

\begin{abstract}
Изучаются трансляционно-инвариантные меры Гиббса для ферромагнитной модели Поттса с $q$ состояниями на дереве Кэли порядка $k$. При этом обобщены некоторые более ранние результаты. Для модели Поттса с тремя состояниями на дереве Кэли порядка $k=3$ исследован вопрос, являются ли некоторые известные трансляционно-инвариантные меры Гиббса крайними или нет.
\end{abstract}

Ключевые слова: дерево Кэли, конфигурация, модель Поттса, мера Гиббса, трансляционно-инвариантные меры, крайность меры.

DOI: https://doi.org/10.4213/tmf9448

\section{1. ВВЕДЕНИЕ}

Известно, что каждой предельной мере Гиббса сопоставляется одна фаза физической системы, поэтому в теории мер Гиббса одной из важных задач является исследование проблемы существования фазового перехода, т. е. изменения состояния физической системы при изменении температуры. Это происходит, когда мера Гиббса неединственна. При этом температура, при которой меняется состояние физической системы, обычно называется критической. Кроме того, известно, что множество всех предельных мер Гиббса образует выпуклое компактное множество [1]-[3]. В связи с этим особый интерес представляет ответ на вопрос, являются ли эти меры крайними.

Для модели Изинга на дереве Кэли [1] существует критическое значение $T_{\mathrm{c}}$ для

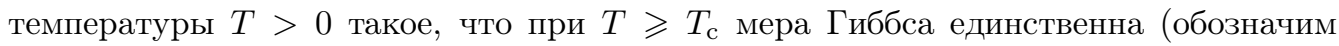
ее через $\left.\mu_{0}\right)$, а при $T<T_{\text {с }}$ существуют две крайние трансляционно-инвариантные меры Гиббса. В работе [4] было доказано, что для модели Изинга на дереве Кэли

${ }^{*}$ Институт математики им. В. И. Романовского Академии наук республики Узбекистан, Ташкент, Узбекистан. E-mail: rozikovu@yandex.ru

${ }^{\dagger}$ Наманганский государственный университет, Наманган, Узбекистан. E-mail: rustam-7102@rambler.ru

${ }^{\ddagger}$ Национальный университет Узбекистана им. Мирзо Улугбека, Ташкент, Узбекистан. E-mail: haydarov_imc@mail.ru 


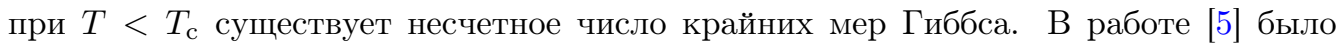
доказано, что мера Гиббса $\mu_{0}$ (неупорядоченное состояние) является крайней тогда и только тогда, когда температура превышает критическую температуру модели спиновых стекол на дереве Кэли.

В статье [6] изучалась ферромагнитная модель Поттса с тремя состояниями на дереве Кэли второго порядка и было показано, что существует критическая темпе-

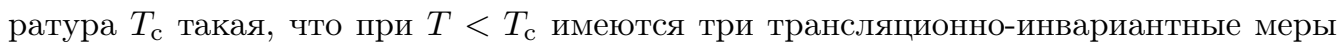
Гиббса и несчетное число мер Гиббса, не являющихся трансляционно-инвариантными. В работе [7] результаты работы [6] были обобщены на случай модели Поттса с конечным числом состояний на дереве Кэли произвольного (конечного) порядка. В работе [8] было показано, что на дереве Кэли трансляционно-инвариантная мера Гиббса антиферромагнитной модели Поттса с внешним полем единственна. В работе [9], посвященой модели Поттса со счетным числом состояний и с ненулевым внешним полем, было доказано, что эта модель имеет единственную трансляционно-инвариантную меру Гиббса.

В работе [10] изучались периодические меры Гиббса, при некоторых условиях было доказано, что все периодические меры Гиббса являются трансляционно-инвариантными; найдены условия, при которых модель Поттса с ненулевым внешним полем имеет периодические меры Гиббса. Работа [11] является продолжением работы [10], в ней было доказано, что в модели Поттса с тремя состояниями и с нулевым внешним полем существуют не менее трех периодических мер Гиббса с периодом два на деревьях Кэли порядка три и четыре. В работе [12] рассматривалась модель Поттса с $q$ состояниями на дереве Кэли порядка $k \geqslant 3$, и на некоторых инвариантах было показано существование периодических (не являющихся трансляционно-инвариантными) мер Гиббса при некоторых условиях на параметры этой модели. Кроме того, была указана нижняя граница количества существующих периодических мер Гиббса. В работе [13] было дано полное описание трансляционно-инвариантных мер Гиббса для ферромагнитной модели Поттса с $q$ состояниями и показано, что их количество равно $2^{q}-1$, а в работе [14] изучалась проблема крайности этих мер. В работе [15] результаты статьи [12] были улучшены и получены явные формулы для трансляционно-инвариантных мер Гиббса для модели Поттса с тремя состояниями на дереве Кэли порядка $k=3$.

В настоящей работе мы обобщаем один из результатов из работы [14]: исследуется проблема крайности трансляционно-инвариантных мер Гиббса для модели Поттса с $q$ состояниями на дереве Кэли порядка $k=2$. Кроме того, мы указываем области, в которых трансляционно-инвариантные меры Гиббса для модели Поттса с тремя состояниями на дереве Кэли порядка $k=3$ являются/не являются крайними.

\section{2. ОПРЕДЕЛЕНИЯ И ИЗВЕСТНЫЕ ФАКТЫ}

Дерево Кэли $\Im^{k}$ порядка $k \geqslant 1$ - это бесконечное дерево, т. е. граф без циклов, из каждой вершины которого выходит ровно $k+1$ ребер. Пусть $\Im^{k}=(V, L, i)$, где $V$ - множество вершин дерева, $L$ - множество его ребер, $i$ - функция инцидентности, которая сопоставляет каждому ребру $l \in L$ его концевые точки $x, y \in V$. Если $i(l)=\{x, y\}$, то $x$ и $y$ называются ближайшими соседями, при этом ребро обозначается как $l=\langle x, y\rangle$. 
Расстояние $d(x, y)$ для $x, y \in V$ на дереве Кэли определяется как минимальное значение $d$, при котором существуют вершины $x=x_{0}, x_{1}, \ldots, x_{d-1}, x_{d}=y$ такие, что $\left\langle x_{0}, x_{1}\right\rangle, \ldots,\left\langle x_{d-1}, x_{d}\right\rangle$ (т. е. вершины последовательно являются ближайшими соседями).

Для фиксированного $x^{0} \in V$ положим

$$
\begin{gathered}
W_{n}=\left\{x \in V \mid d\left(x, x^{0}\right)=n\right\}, \quad V_{n}=\left\{x \in V \mid d\left(x, x^{0}\right) \leqslant n\right\}, \\
L_{n}=\left\{l=\langle x, y\rangle \in L \mid x, y \in V_{n}\right\} .
\end{gathered}
$$

Известно, что существует взаимно однозначное соответствие между множеством $V$ вершин дерева Кэли порядка $k \geqslant 1$ и группой $G_{k}$, являющейся свободным произведением $k+1$ циклических групп второго порядка с образующими $a_{1}, a_{2}, \ldots, a_{k+1}$ соответственно.

Рассмотрим модель, в которой спиновые переменные принимают значения из множества $\Phi=\{1,2, \ldots, q\}, q \geqslant 2$, и расположены в вершинах дерева. Тогда конфигурация $\sigma$ на $V$ определяется как функция $x \in V \rightarrow \sigma(x) \in \Phi$; множество всех конфигураций совпадает с $\Omega=\Phi^{V}$.

Гамильтониан модели Поттса определяется как

$$
H(\sigma)=-J \sum_{\langle x, y\rangle \in L} \delta_{\sigma(x) \sigma(y)}
$$

где $J \in \mathbb{R},\langle x, y\rangle$ - ближайшие соседи и $\delta_{i j}-$ символ Кронекера. Определим конечномерное распределение вероятностной меры $\mu_{n}$ в обьеме $V_{n}$ :

$$
\mu_{n}\left(\sigma_{n}\right)=Z_{n}^{-1} \exp \left\{-\beta H_{n}\left(\sigma_{n}\right)+\sum_{x \in W_{n}} \tilde{h}_{\sigma(x), x}\right\},
$$

где $\beta=1 / T, T>0$ - температура, $Z_{n}^{-1}$ - нормировочный множитель,

$$
H_{n}\left(\sigma_{n}\right)=-J \sum_{\langle x, y\rangle \in L_{n}} \delta_{\sigma(x) \sigma(y)}
$$

и $\left\{\tilde{h}_{x}=\left(\tilde{h}_{1, x}, \ldots, \tilde{h}_{q, x}\right) \in \mathbb{R}^{q}, x \in V\right\}-$ совокупность векторов.

Говорят, что последовательность вероятностных распределений (2.2) согласованная, если для всех $n \geqslant 1$ и $\sigma_{n-1} \in \Phi^{V_{n-1}}$

$$
\sum_{\omega_{n} \in \Phi^{W_{n}}} \mu_{n}\left(\sigma_{n-1} \vee \omega_{n}\right)=\mu_{n-1}\left(\sigma_{n-1}\right),
$$

где $\sigma_{n-1} \vee \omega_{n}$ - объединение конфигураций. В этом случае существует единственная мера $\mu$ на $\Phi^{V}$ такая, что для всех $n$ и $\sigma_{n} \in \Phi^{V_{n}}$

$$
\mu\left(\left\{\left.\sigma\right|_{V_{n}}=\sigma_{n}\right\}\right)=\mu_{n}\left(\sigma_{n}\right) .
$$

Такая мера называется расщепленной мерой Гиббса, соответствующей гамильтониану (2.1) и векторнозначной функции $\tilde{h}_{x}, x \in V$. Следующее утверждение [8] описывает условие для $\tilde{h}_{x}$, обеспечивающее согласованность меры $\mu_{n}\left(\sigma_{n}\right)$. 
ТЕОрема 1. Вероятностные распределения $\mu_{n}\left(\sigma_{n}\right), n=1,2, \ldots$, заданные в $(2.2)$, являются согласованными тогда и только тогда, когда для любого $x \in V$ имеет место следующее равенство:

$$
h_{x}=\sum_{y \in S(x)} F\left(h_{y}, \theta\right),
$$

где функиия $F: h=\left(h_{1}, \ldots, h_{q-1}\right) \in \mathbb{R}^{q-1} \rightarrow F(h, \theta)=\left(F_{1}, \ldots, F_{q-1}\right) \in \mathbb{R}^{q-1}$ определяется формулами

$$
F_{i}=\ln \frac{(\theta-1) e^{h_{i}}+\sum_{j=1}^{q-1} e^{h_{j}}+1}{\theta+\sum_{j=1}^{q-1} e^{h_{j}}}, \quad \theta=e^{J \beta},
$$

$S(x)$ - множество прямых потомков точки $x u h_{x}=\left(h_{1, x}, \ldots, h_{q-1, x}\right)$ удовлетворяет условию

$$
h_{i, x}=\tilde{h}_{i, x}-\tilde{h}_{q, x}, \quad i=1, \ldots, q-1 .
$$

Трансляционно-инвариантная расщепленная мера Гиббса (ТИРМГ) соответствует решению $h_{x}$ уравнения $(2.3)$ с $h_{x}=h=\left(h_{1}, \ldots, h_{q-1}\right) \in \mathbb{R}^{q-1}$ для всех $x \in V$. Тогда из уравнения (2.3) получим $h=k F(h, \theta)$ и, положив $z_{i}=e^{h_{i}}, i=1, \ldots, q-1$, можем переписать последнее уравнение как

$$
z_{i}=\left(\frac{(\theta-1) z_{i}+\sum_{j=1}^{q-1} z_{j}+1}{\theta+\sum_{j=1}^{q-1} z_{j}}\right)^{k}, \quad i=1, \ldots, q-1 .
$$

В работе [13] были получены следующие результаты.

1. Путем решения уравнения (2.5) полностью описано множество ТИРМГ и показано, что любая ТИРМГ модели Поттса соответствует решению уравнения

$$
z=f_{m}(z) \equiv\left(\frac{(\theta+m-1) z+q-m}{m z+q-m-1+\theta}\right)^{k}
$$

для некоторого $m=1 \ldots,[q / 2]$.

2. Показано, что существуют значения $\theta_{m}=\theta_{m}(k)$, которые являются критическими для изменения количества ТИРМГ (при $k=2$ имеется явная формула $\left.\theta_{m}(2)=1+2 \sqrt{m(q-m)}\right), m=1, \ldots,[q / 2] ;$ если $\theta<\theta_{1}$, то при $k \geqslant 2, J>0$ существует единственная ТИРМГ.

\section{3. УСЛОВИЯ КРАЙНОСТИ МЕР}

Следуя работе [14], чтобы проверить, является ли мера крайней, мы используем величины из теории реконструкции на деревьях [16]-[18].

Для каждого фиксированного $m$ уравнение (2.6) имеет не более трех решений: $z_{0}=1, z_{i}=z_{i}(\theta, q, m), i=1,2$, причем $z_{1}<z_{2}$ (см. шаг 1 доказательства теоремы 1 из работы [13]). Обозначим через $\mu_{i}=\mu_{i}(\theta, m)$ ТИРМГ модели Поттса, которая соответствует решению $z_{i}$.

Для $l=(\underbrace{z, \ldots, z}_{m}, \underbrace{1, \ldots, 1}_{q-m})$ ТИРМГ, соответствующая вектору $l \in \mathbb{R}^{q}$, является цепью Маркова [14] с состояниями $\{1, \ldots, q\}$ и матрицей переходных вероятностей 
$\mathbb{P}=\left(P_{i j}\right)$ с элементами

$$
P_{i j}=\frac{l_{j} e^{J \beta \delta_{i j}}}{\sum_{r=1}^{q} l_{r} e^{J \beta \delta_{i r}}} .
$$

Из этого выражения получаем

$$
P_{i j}=\left\{\begin{array}{lll}
\theta z / Z_{1}, & \text { если } \quad i=j, i \in\{1, \ldots, m\}, \\
z / Z_{1}, & \text { если } \quad i \neq j, i, j \in\{1, \ldots, m\}, \\
1 / Z_{1}, & \text { если } \quad i \in\{1, \ldots, m\}, j \in\{m+1, \ldots, q\}, \\
z / Z_{2}, & \text { если } \quad i \in\{m+1, \ldots, q\}, j \in\{1, \ldots, m\}, \\
\theta / Z_{2}, & \text { если } i=j, i \in\{m+1, \ldots, q\}, \\
1 / Z_{2}, & \text { если } i \neq j, i, j \in\{m+1, \ldots, q\},
\end{array}\right.
$$

где

$$
Z_{1}=(\theta+m-1) z+q-m, \quad Z_{2}=m z+\theta+q-m-1 .
$$

Приведем необходимые определения из работы [19]. Рассмотрим конечное полное поддерево $\mathcal{T}$, содержащее все начальные точки полудерева $\Gamma_{x^{0}}^{k}$. Граница $\partial \mathcal{T}$ поддерева $\mathcal{T}$ состоит из ближайших соседей его вершин, которые лежат в $\Gamma_{x^{0}}^{k} \backslash \mathcal{T}$. Мы отождествляем поддерево $\mathcal{T}$ с множеством его вершин. Через $E(A)$ обозначим множество всех ребер $A$ и $\partial A$.

В работе [19] ключевыми являются величины $\kappa$ и $\gamma$. Эти параметры определяют свойства множества мер Гиббса $\left\{\mu_{\mathcal{T}}^{\tau}\right\}$, где граничное условие $\tau$ фиксировано, а $\mathcal{T}$ является произвольным начальным полным и конечным поддеревом в $\Gamma_{x^{0}}^{k}$. Для данного начального поддерева $\mathcal{T}$ дерева $\Gamma_{x^{0}}^{k}$ и вершины $x \in \mathcal{T}$ мы будем писать $\mathcal{T}_{x}$ для (максимального) поддерева $\mathcal{T}$ с начальной точкой в $x$. Когда $x$ не является начальной точкой поддерева $\mathcal{T}$, через $\mu_{\mathcal{T}_{x}}^{s}$ обозначим меру Гиббса, в которой "предок" $x$ имеет спин $s$, а конфигурация на нижней границе $\mathcal{T}_{x}$ (т. е. на $\partial \mathcal{T}_{x} \backslash\{x\}$ ) задается через $\tau$.

Для двух мер $\mu_{1}$ и $\mu_{2}$ на $\Omega$ введем расстояние

$$
\left\|\mu_{1}-\mu_{2}\right\|_{x}=\frac{1}{2} \sum_{i=1}^{q}\left|\mu_{1}(\sigma(x)=i)-\mu_{2}(\sigma(x)=i)\right| .
$$

Пусть $\eta^{x, s}$ есть конфигурация $\eta$ со спином в $x$, равным $s$.

Следуя работе [19], определим величины

$$
\begin{aligned}
& \kappa \equiv \kappa(\mu)=\sup _{x \in \Gamma^{k}} \max _{x, s, s^{\prime}}\left\|\mu_{\mathcal{T}_{x}}^{s}-\mu_{\mathcal{T}_{x}}^{s^{\prime}}\right\|_{x}, \\
& \gamma \equiv \gamma(\mu)=\sup _{A \subset \Gamma^{k}} \max _{\eta, y, x, s, s^{\prime}}\left\|\mu_{A}^{\eta^{y, s}}-\mu_{A}^{\eta^{y, s^{\prime}}}\right\|_{x},
\end{aligned}
$$

где максимум берется по всем граничным условиям $\eta$, всем $y \in \partial A$, всем соседям $x \in A$ вершины $y$ и всем спинам $s, s^{\prime} \in\{1, \ldots, q\}$. Заметим, что $\kappa$ имеет простой вид [19]:

$$
\kappa=\frac{1}{2} \max _{i, j} \sum_{l}\left|P_{i l}-P_{j l}\right| .
$$


Для величины $\gamma$ общего выражения не существует, но в специфических моделях (таких, как модели Изинга, модель Hard Core и т. д.) ее можно оценить. Например, если $\mathbb{P}$ - симметрическая матрица модели Поттса (т. е. матрица, соответствующая решению $z=1$ ), то $\gamma \leqslant \frac{\theta-1}{\theta+1}[19]$.

Достаточным условием того, что мера Гиббса $\mu$ крайняя, является неравенство (см. теорему 9.3 в [19])

$$
k \kappa(\mu) \gamma(\mu)<1 .
$$

Используя формулы (3.2) для $i \neq j$ и (3.4), получим [14]

$$
\frac{1}{2} \sum_{l=1}^{q}\left|P_{i l}-P_{j l}\right|= \begin{cases}a, & \text { если } i, j=1, \ldots, m, \\ b, & \text { если } i, j=m+1, \ldots, q, \\ c & \text { в остальных случаях, }\end{cases}
$$

где $a, b$ и $c$ определяются как

$$
\begin{gathered}
a=\frac{(\theta-1) z}{Z_{1}}, \quad b=\frac{(\theta-1) \sqrt[k]{z}}{Z_{1}}, \\
c=\frac{1}{2 Z_{1}}(z|\theta-\sqrt[k]{z}|+|1-\theta \sqrt[k]{z}|+(z(m-1)+q-m-1)|1-\sqrt[k]{z}|),
\end{gathered}
$$

a $Z_{1}, Z_{2}$ заданы в (3.3). Ясно, что

$$
\kappa= \begin{cases}\max \{b, c\}, & \text { если } m=1, \\ \max \{a, b, c\}, & \text { если } m \geqslant 2 .\end{cases}
$$

Рассмотрим случай $z \neq 1$ (где $z=x^{2}$ и $x$ есть решения уравнения (2.6)). Возьмем ющая матрица есть $\mathbb{P}$.

\section{4. ПРОБЛЕМА КРАЙНОСТИ ТРАНСЛЯЦИОННО-ИНВАРИАНТНЫХ МЕР ПРИ $k=2$}

Известна следующая теорема [14].

Teоpema 2. Пусть $k=2$.

1. Если $m=2$, то для каждого $q=4,5,6,7,8$ существует $\breve{\theta}>\theta_{\mathrm{c}}=q+1$ такое, что мера $\mu_{1}(\theta, 2)$ является крайней при любых $\theta \in\left[\theta_{2}, \breve{\theta}\right) ;$ для каждого $q \geqslant 9$ существует $\theta^{\dagger} \in\left(\theta_{2}, q+1\right)$ такое, что мера $\mu_{1}(\theta, 2)$ является крайней при любых $\theta \in\left[\theta^{\dagger}, \breve{\theta}\right)$. Число $\theta^{\dagger}=\theta^{\dagger}(q)$ является единственным решением уравнения

$$
\theta^{3}-(q+3) \theta^{2}+(6 q-17) \theta-(9 q-19)=0,
$$

а $\breve{\theta}=\breve{\theta}(q)$ есть единственное решение уравнения

$$
\theta^{3}-(q+3) \theta^{2}-(2 q-15) \theta-(q+13)=0 .
$$

2. Если $m=2$, то для каждого $q=4,5,6,7,8$ существует $\grave{\theta}=\grave{\theta}(q)$ mакое, что $\theta_{2}<\grave{\theta} \leqslant q+1$ и $\mu_{2}(\theta, 2)$ является крайней при любых $\theta \in\left[\theta_{2}, \grave{\theta}\right)$.

3. Если $q<\frac{m+1}{2 m}\left[3 m+1+\sqrt{m^{2}+6 m+1}\right]$ u $m \geqslant 2$, то мера $\mu_{1}\left(\theta_{m}, m\right)=\mu_{2}\left(\theta_{m}, m\right)$ является крайней. 
Следующая теорема является обобщением теоремы 2.

Теорема 3. Пусть $k=2, m \geqslant 2$. Тогда верны следующие утверждения.

1. Если $2 m \leqslant q<\frac{m+1}{2 m}\left[3 m+1+\sqrt{m^{2}+6 m+1}\right]$, то существует $\ddot{\theta}>q+1$ такое, что мера $\mu_{1}(\theta, m)$ является крайней при любъх $\theta \in\left[\theta_{m}, \ddot{\theta}\right)$.

2. Если $q>\frac{m+1}{2 m}\left[3 m+1+\sqrt{m^{2}+6 m+1}\right]$, то существует $\bar{\theta} \in\left(\theta_{m}, \theta_{\mathrm{c}}\right)$ такое, что мера $\mu_{1}(\theta, m)$ является крайней при любых $\theta \in(\bar{\theta}, \ddot{\theta})$.

3. Если $2 m \leqslant q<m+\frac{1}{4 m}\left(m+1+\sqrt{m^{2}+2 m+7}\right)^{2}=\zeta(m)$, то существует $\overline{\bar{\theta}} \in\left(\theta_{m},+\infty\right)$ такое, что мера $\mu_{2}(\theta, m)$ является крайней при любых $\theta \in\left[\theta_{m}, \overline{\bar{\theta}}\right)$.

ДокАЗАтЕльство. 1. Положим $\frac{m+1}{2 m}\left[3 m+1+\sqrt{m^{2}+6 m+1}\right]=\alpha(m)$. Проверим крайность меры $\mu_{1}(\theta, m)$ при $z_{1} \geqslant 1$. В этом случае $\theta \in\left[\theta_{m}, \theta_{c}\right]$. Тогда нетрудно показать, что $\kappa=a$. Следовательно, мы должны проверить выполнение неравенства

$$
2 \kappa \gamma \leqslant 2 a \frac{\theta-1}{\theta+1}<1
$$

Поскольку

$$
\sqrt{z_{1}}=\frac{2(q-m)}{\theta-1+\sqrt{(\theta-1)^{2}-4 m(q-m)}},
$$

мы получим неравенство

$$
\theta^{2}-(2 m+4) \theta-2 m+3-(\theta-3) \sqrt{(\theta-1)^{2}-4 m(q-m)}<0,
$$

которое эквивалентно неравенству

$$
u(\theta)=\theta^{3}-(q+3) \theta^{2}+(8 q-10 m-1) \theta-(9 q-8 m-12)>0 .
$$

Заметим, что $u\left(\theta_{m}\right)>0$ при $q<\alpha(m)$. Кроме того, функция $u(\theta)$ возрастает на множестве $\left[\theta_{m}^{(1)},+\infty\right]$, где

$$
\theta_{m}^{(1)}=\frac{q+3+\sqrt{(q+3)^{2}-3(8 q-10 m-1)}}{3} .
$$

Из неравенства $[q / 2] \geqslant m$ следует, что $\theta_{m}^{(1)} \leqslant \theta_{m}$. Действительно, рассмотрим неравенство

$$
\frac{q+3+\sqrt{(q+3)^{2}-3(8 q-10 m-1)}}{3} \leqslant 1+2 \sqrt{m(q-m)},
$$

которое эквивалентно неравенству

$$
\xi(q)=2 q \sqrt{m(q-m)}+5 m+2-3 q-6 m(q-m) \leqslant 0 .
$$

Вычислим производную

$$
\xi^{\prime}(q)=2 \sqrt{m(q-m)}+\frac{m q}{\sqrt{m(q-m)}}-3-6 m .
$$

Функция $\xi(q)$ возрастает при $m \leqslant\left[\frac{q}{2}\right]$ и $q<\frac{m+1}{2 m}\left[3 m+1+\sqrt{m^{2}+6 m+1}\right]$. Из последнего неравенства имеем

$$
q<\frac{2(m+1)^{2}}{m} \leqslant 2(m+3) .
$$


Отсюда $\xi(q)<\xi(2(m+3))$. Далее, легко увидеть, что $\xi(2(m+3))<0$ при $m \geqslant 2$ и $\theta_{m}^{(1)} \leqslant \theta_{m}$. Поэтому функция $u(\theta)$ возрастает на отрезке $\left[\theta_{m}, \theta_{\mathrm{c}}\right]$, т. е. $u(\theta)>0$ при любых $\theta \in\left[\theta_{m}, \theta_{c}\right]$. Следовательно, мера $\mu_{1}(\theta, m)$ является крайней, если $z_{1}>1$ и $\theta \in\left[\theta_{m}, \theta_{\mathrm{c}}\right]$.

Пусть $z_{1}<1$. В этом случае независимо от значений $q$ и $m$ имеем

$$
c=\frac{1}{Z_{1}}\left(\theta \sqrt{z_{1}}-1\right) .
$$

Легко увидеть, что $b \geqslant c$ при $z_{1}<1$, т. е. при $\theta>\theta_{\text {c. }}$ Следовательно, $\kappa=b$ и мы можем проверить, что

$$
2 \kappa \gamma \leqslant \frac{2(\theta-1) \sqrt{z_{1}}}{\theta z_{1}+q-1} \cdot \frac{\theta-1}{\theta+1}<1 .
$$

Положим

$$
g(\theta, q, m)=\frac{2(\theta-1) \sqrt{z_{1}}}{\theta z_{1}+q-1} \cdot \frac{\theta-1}{\theta+1}-1
$$

Из уравнения

$$
\sqrt{z_{1}}=\frac{2(q-m)}{\theta-1+\sqrt{(\theta-1)^{2}-4 m(q-m)}}
$$

имеем

$$
g\left(\theta_{\mathrm{c}}, q, m\right)=\frac{\theta-1}{\theta+1}-1<0, \quad \lim _{\theta \rightarrow+\infty} g(\theta, q, m)=\frac{q-2 m+1}{q-1}>0 .
$$

Таким образом, существует $\theta^{\prime \prime} \in\left(\theta_{\mathrm{c}},+\infty\right)$ такое, что $g\left(\theta^{\prime \prime}, q, m\right)=0$. Пусть

$$
\ddot{\theta}=\min \left\{\theta^{\prime \prime}: g\left(\theta^{\prime \prime}, q, m\right)=0\right\} .
$$

Тогда $g(\theta, q, m)<0$ при любых $\theta \in\left[\theta_{c}, \ddot{\theta}\right)$, т. е. при этом условии мера $\mu_{1}(\theta, m)$ является крайней.

2. Пусть $z_{1} \geqslant 1$. Если $q>\frac{m+1}{2 m}\left[3 m+1+\sqrt{m^{2}+6 m+1}\right]$, то $u\left(\theta_{m}\right)<0$. Заметим, что $u\left(\theta_{\mathrm{c}}\right)>0$. Кроме того, функция $u(\theta)$ возрастает при $\left[\theta_{m}, \theta_{\mathrm{c}}\right]$. Следовательно, существует единственное $\bar{\theta} \in\left(\theta_{m}, \theta_{\mathrm{c}}\right]$ такое, что $u(\bar{\theta})=0$. Из условия крайности (4.1) получим, что мера $\mu_{1}(\theta, m)$ является крайней при $\theta \in\left(\bar{\theta}, \theta_{\mathrm{c}}\right)$.

В случае $z_{1}<1$ аналогично доказательству п. 1 этой теоремы можно получить, что мера $\mu_{1}(\theta, m)$ является крайней при $\theta \in(\bar{\theta}, \ddot{\theta})$.

3. При $\theta \geqslant \theta_{m}$ имеем $z_{2} \geqslant 1$. Проверим условие крайности меры $\mu_{2}(\theta, m)$ :

$$
2 \kappa \gamma \leqslant 2 c \frac{\theta-1}{\theta+1}<1 .
$$

Оно эквивалентно неравенству

$$
v(\theta)=\theta^{2}-(2 m+4) \theta-2 m+3+(\theta-3) \sqrt{(\theta-1)^{2}-4 m(q-m)}<0,
$$

которое имеет решение, если имеет решение неравенство

$$
\theta^{2}-(2 m+4) \theta-(2 m-3)<0 \text {. }
$$




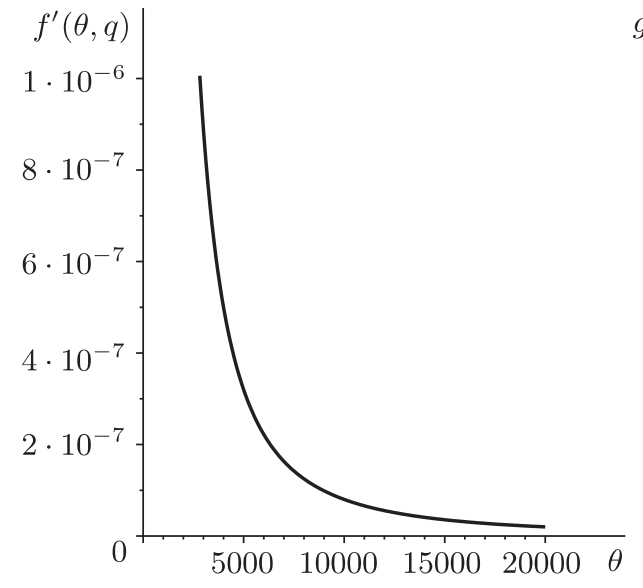

a

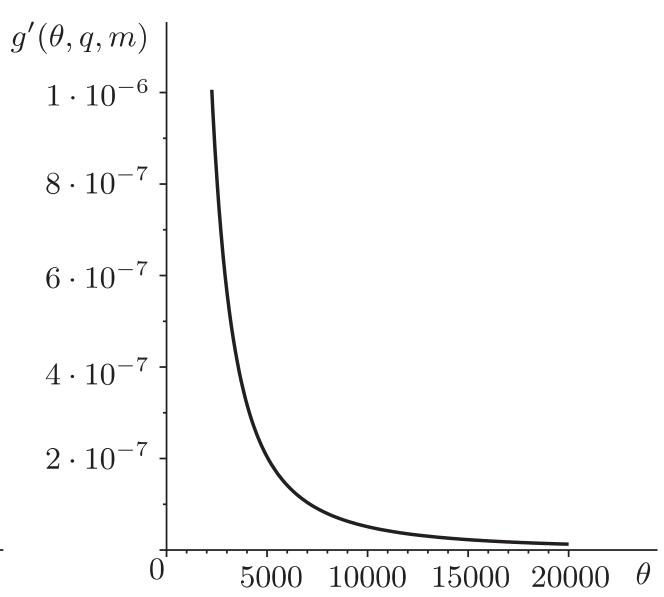

б

Рис. 1. График функции $f^{\prime}(\theta, q)$ при $q=5, m=1$ (а) и график функции $g^{\prime}(\theta, q, m)$ при $q=8, m=3$ (б).

Решение последнего неравенства таково:

$$
\theta<m+2+\sqrt{m^{2}+2 m+7}
$$

Так как $\theta \geqslant \theta_{m}=1+2 \sqrt{m(q-m)}$, получим

$$
1+2 \sqrt{m(q-m)}<m+2+\sqrt{m^{2}+2 m+7} .
$$

Отсюда

$$
2 m \leqslant q<m+\frac{1}{4 m}\left(m+1+\sqrt{m^{2}+2 m+7}\right)^{2} .
$$

Легко увидеть, что при этом условии

$$
\lim _{\theta \rightarrow+\infty} v(\theta)=+\infty
$$

Следовательно, существует $\overline{\bar{\theta}} \in\left(\theta_{m},+\infty\right)$ такое, что $v(\overline{\bar{\theta}})=0$. Таким образом, мера $\mu_{2}(\theta, m)$ является крайней при $\theta \in\left(\theta_{m}, \overline{\bar{\theta}}\right)$. Теорема доказана.

ЗАмЕЧАниЕ 1 . Если $q>m+\frac{1}{4 m}\left(m+1+\sqrt{m^{2}+2 m+7}\right)^{2}$, то условие крайности (4.3) не выполняется ни при каких $\theta$, т. е. в этом случае неизвестно, является ли мера $\mu_{2}(\theta, m)$ крайней.

ЗАмечАниЕ 2. Графически можно показать, что при конечных $q$ и $m$ производные $f^{\prime}(\theta, q)>0, g^{\prime}(\theta, q, m)>0$ (см. рис. 1$)$, т. е. функции $f(\theta, q)$ и $g(\theta, q, m)$ возрастают (доказать это аналитически очень трудно). Следовательно, величины $\theta^{\prime}, \theta^{\prime \prime}$ определены единственным образом, $\tilde{\theta}=\theta^{\prime}, \ddot{\theta}=\theta^{\prime \prime}$. 


\section{5. ПРОБЛЕМА КРАЙНОСТИ ТРАНСЛЯЦИОННО-ИНВАРИАНТНЫХ МЕР ПРИ $k=3$}

Пусть

$$
\begin{gathered}
\theta_{\mathrm{c}}=\sqrt{9+6 \sqrt{3}}-2, \\
p(\theta)=-\frac{1}{3}\left(\theta^{2}+\theta-2\right), \quad r(\theta)=\frac{1}{27}\left(-2(\theta-1)^{3}-9(\theta-1)^{2}+54\right), \\
\alpha(\theta)=\operatorname{arctg}\left(\frac{2}{r} \sqrt{-\frac{r^{2}}{4}-\frac{p^{3}}{27}}\right), \quad r(\theta) \neq 0, \\
x_{1}(\theta)=\frac{2 p}{3} \cos \frac{\alpha+2 \pi}{3}+\frac{\theta-1}{3}>0, \quad x_{2}(\theta)=\frac{2 p}{3} \cos \frac{\alpha+4 \pi}{3}+\frac{\theta-1}{3}>0, \\
x_{3}=\frac{1}{2}\left(-1+\sqrt{3}+\frac{1}{3} \sqrt{9+6 \sqrt{3}}(3-\sqrt{3})\right) .
\end{gathered}
$$

Известно следующее утверждение [15].

Теорема 4. Пусть $q=3, k=3, \theta>1$. Тогда система уравнений (2.5) имеет:

1) единственное решение $(1,1)$ при $\theta<\theta_{c}$;

2) три решения $(1,1),\left(z_{1}, 1\right),\left(1, z_{1}\right)$ при $\theta=\theta_{c}$;

3) семь решений $(1,1),\left(z_{2}, 1\right),\left(1, z_{2}\right),\left(z_{3}, 1\right),\left(1, z_{3}\right),\left(z_{2}, z_{2}\right),\left(z_{3}, z_{3}\right)$ при $\theta>\theta_{\mathrm{c}}$, әде $z_{1}=x_{3}^{3}, z_{2}=x_{1}^{3}, z_{3}=x_{2}^{3}$.

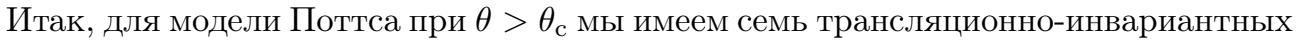
мер Гиббса. Заметим, что в силу симметрии область, где являются крайними меры, соответствующие решениям $\left(z_{2}, 1\right)$ и $\left(1, z_{2}\right)$ (и аналогично меры, соответствующие решениям $\left(z_{3}, 1\right)$ и $\left.\left(1, z_{3}\right)\right)$, одна и та же. Поэтому в следующем пункте мы исследуем, являются ли крайними меры $\mu_{0}, \mu_{1}, \mu_{2}, \mu_{3}, \mu_{4}$, соответствующие решениям $(1,1)$, $\left(z_{2}, 1\right),\left(z_{3}, 1\right),\left(z_{2}, z_{2}\right)$ и $\left(z_{3}, z_{3}\right)$.

Для мер $\mu_{0}, \mu_{1}, \mu_{2}$ матрица переходных вероятностей имеет вид

$$
\mathbb{P}_{1}=\left(\begin{array}{ccc}
\theta z / Z_{1} & 1 / Z_{1} & 1 / Z_{1} \\
z / Z_{2} & \theta / Z_{2} & 1 / Z_{2} \\
z / Z_{2} & 1 / Z_{2} & \theta / Z_{2}
\end{array}\right),
$$

где $Z_{1}=\theta z+2, Z_{2}=z+\theta+1$. Кроме того, $\kappa=\max \{b, c\}$, где

$$
b=\frac{(\theta-1) \sqrt[3]{z}}{Z_{1}}, \quad c=\frac{1}{2 Z_{1}}(z|\theta-\sqrt[3]{z}|+|1-\theta \sqrt[3]{z}|+|1-\sqrt[3]{z}|) .
$$

Для мер $\mu_{3}, \mu_{4}$ матрица переходных вероятностей имеет вид

$$
\mathbb{P}_{2}=\left(\begin{array}{ccc}
\theta z / Z_{1}^{\prime} & z / Z_{1}^{\prime} & 1 / Z_{1}^{\prime} \\
z / Z_{1}^{\prime} & \theta z / Z_{1}^{\prime} & 1 / Z_{1}^{\prime} \\
z / Z_{2}^{\prime} & z / Z_{2}^{\prime} & \theta / Z_{2}^{\prime}
\end{array}\right)
$$

где $Z_{1}^{\prime}=(\theta+1) z+1, Z_{2}^{\prime}=2 z+\theta$. Кроме того, $\kappa=\max \left\{a^{\prime}, b^{\prime}, c^{\prime}\right\}$, где

$$
a^{\prime}=\frac{(\theta-1) z}{Z_{1}^{\prime}}, \quad b^{\prime}=\frac{(\theta-1) \sqrt[3]{z}}{Z_{1}^{\prime}}, \quad c^{\prime}=\frac{1}{2 Z_{1}^{\prime}}(z|\theta-\sqrt[3]{z}|+|1-\theta \sqrt[3]{z}|+z|1-\sqrt[3]{z}|) .
$$


5.1. Условия, при которых мера не является крайней. Как известно, чтобы мера Гиббса $\mu$, соответствующая $\mathbb{P}$, не являлась крайней, достаточно выполнения неравенства $k \lambda_{2}^{2}>1$, где $\lambda_{2}$ есть второе по абсолютной величине собственное значение матрицы $\mathbb{P}$ (достаточное условие Кестена-Стигума) [17].

Рассмотрим меры $\mu_{i}, i=0,1, \ldots, 4$. Из соотношений $(5.1),(5.2)$, решив уравнения $\operatorname{det}\left(\mathbb{P}_{1}-\lambda E\right)=0, \operatorname{det}\left(\mathbb{P}_{2}-\lambda^{\prime} E\right)=0$, найдем собственные значения матриц $\mathbb{P}_{1}$ и $\mathbb{P}_{2}$. Они имеют соответственно вид

$$
\begin{array}{llll}
\lambda_{1}=1, & \lambda_{2}=\frac{\theta-1}{z+\theta+1}, & \lambda_{3}=\frac{\left(\theta^{2}+\theta-2\right) z}{(\theta z+2)(z+\theta+1)}, \\
\lambda_{1}^{\prime}=1, & \lambda_{2}^{\prime}=\frac{(\theta-1) z}{(\theta+1) z+1}, & \lambda_{3}^{\prime}=\frac{\left(\theta^{2}+\theta-2\right) z}{(2 z+\theta)[(\theta+1) z+1]} .
\end{array}
$$

При этом

$$
\max \left\{\left|\lambda_{2}\right|,\left|\lambda_{3}\right|\right\}=\left\{\begin{array}{lll}
\lambda_{2}, & \text { если } & z<1, \\
\lambda_{3}, & \text { если } & z>1 ;
\end{array} \quad \max \left\{\left|\lambda_{2}^{\prime}\right|,\left|\lambda_{3}^{\prime}\right|\right\}= \begin{cases}\lambda_{2}^{\prime}, & \text { если } z>1, \\
\lambda_{3}^{\prime}, & \text { если } \quad z<1 .\end{cases}\right.
$$

При $z=1$ матрица (5.1) имеет собственные значения

$$
\lambda_{1}=1, \quad \lambda \equiv \lambda_{2}=\lambda_{3}=\frac{\theta-1}{\theta+2} .
$$

Проверим условие $3 \lambda^{2}>1$, при выполнении которого единственная мера $\mu_{0}$ не является крайней. Решим неравенство $3\left(\frac{\theta-1}{\theta+2}\right)^{2}>1$, получим $\theta>\frac{1}{2}(5+3 \sqrt{3})$. Таким образом, мера $\mu_{0}$ не является крайней при $\theta>\frac{1}{2}(5+3 \sqrt{3}) \approx 5.0981$.

Теперь проверим условие, при котором меры $\mu_{1}, \mu_{3}$ не являются крайними. Так как явный вид функции $z_{2}(\theta)$ очень громоздкий, воспользуемся компьютерным анализом. С помощью программы Maple мы получили, что $z_{2}(\theta)>1$ при $\theta_{\text {c }}<\theta \leqslant \hat{\theta}$ и $\theta>\check{\theta} \approx 3.339149$ и $z_{2}(\theta)<1$ при $\hat{\theta}<\theta<\check{\theta}$, где

$$
\hat{\theta}=\frac{3}{2} \sqrt[3]{3+2 \sqrt{2}}+\frac{3}{2 \sqrt[3]{3+2 \sqrt{2}}}-\frac{1}{2} \approx 3.032952097
$$

Следовательно, для меры $\mu_{1}$ при $z_{2}(\theta)>1$ и $z_{2}(\theta)<1$ требуется проверить условия $3 \lambda_{3}^{2}>1$ и $3 \lambda_{2}^{2}>1$ соответственно. Оба последних неравенства не имеют решений (см. рис. 2), т. е. мера $\mu_{1}$ заведомо является крайней. Далее, для меры $\mu_{3}$ при $z_{2}(\theta)>1$ и $z_{2}(\theta)<1$ требуется проверить условия $3\left(\lambda_{2}^{\prime}\right)^{2}>1$ и $3\left(\lambda_{3}^{\prime}\right)^{2}>1$ соответственно. Первое неравенство имеет решение $\theta>\theta^{(0)} \approx 3.994$, а второе не имеет решений (см. рис. 3), т. е. мера $\mu_{3}$ не является крайней при $\theta>\theta^{(0)}$.

Проверим условие, при котором не являются крайними меры $\mu_{2}, \mu_{4}$, соответствующие вектор-функциям $\left(z_{3}(\theta), 1\right)$ и $\left(z_{3}(\theta), z_{3}(\theta)\right)$. Заметим, что $z_{3}(\theta)$ имеет громоздкий вид. С помощью компьютерного анализа можно увидеть, что $z_{3}(\theta)>1$ при $\theta_{\text {с }}<\theta<\theta^{(1)} \approx 2.444, \theta \geqslant \hat{\theta}$ и $z_{3}(\theta)<1$ при $\theta^{(1)}<\theta<\hat{\theta}$. Следовательно, для меры $\mu_{2}$ при $z_{3}(\theta)>1$ и $z_{3}(\theta)<1$ требуется проверить условия $3 \lambda_{3}^{2}>1$ и $3 \lambda_{2}^{2}>1$ соответственно. Оба последних неравенства не имеют решений (см. рис. 4), т. е. мера $\mu_{2}$ заведомо является крайней. Далее, для меры $\mu_{4}$ при $z_{3}(\theta)>1$ и $z_{3}(\theta)<1$ рассмотрим соответственно неравенства $3\left(\lambda_{2}^{\prime}\right)^{2}>1$ и $3\left(\lambda_{3}^{\prime}\right)^{2}>1$. Первое неравенство имеет решение вида $\theta>\theta^{(2)} \approx 3.759659$, а второе не имеет решения (см. рис. 5$)$. Следовательно, мера $\mu_{4}$ не является крайней при $\theta>\theta^{(2)}$. 


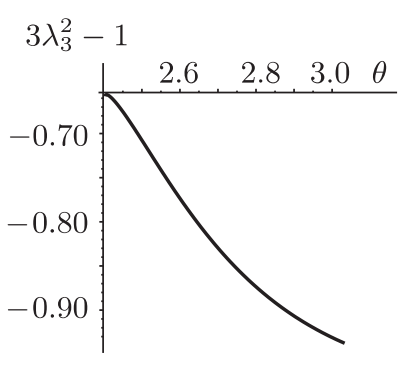

a

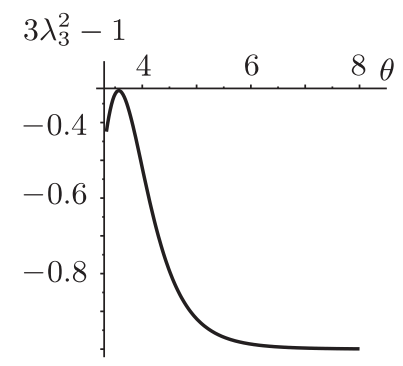

б

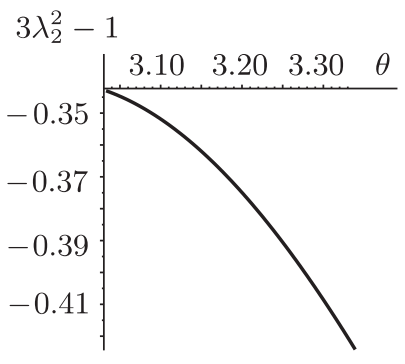

B

Рис. 2. График функции $3 \lambda_{3}^{2}-1$ при $\theta_{\mathrm{c}}<\theta \leqslant \hat{\theta}(\mathrm{a})$, при $\theta>\check{\theta}$ (б) и график функции $3 \lambda_{2}^{2}-1$ при $\hat{\theta}<\theta<\check{\theta}$ (в).

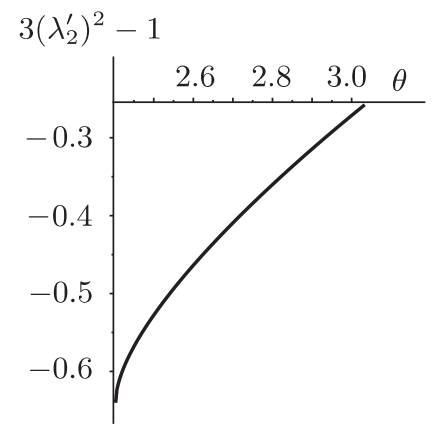

a

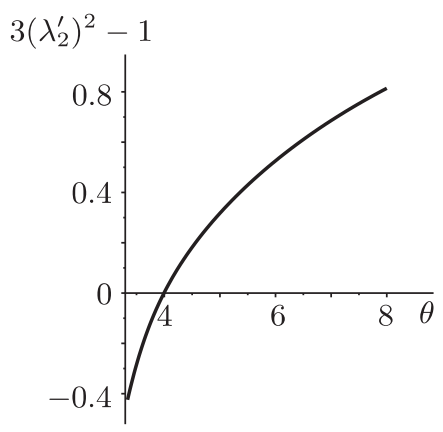

б

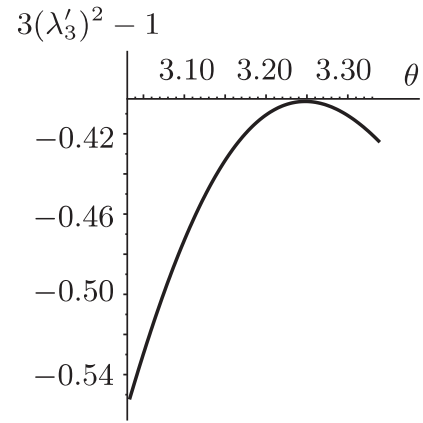

B

Рис. 3. График функции $3\left(\lambda_{2}^{\prime}\right)^{2}-1$ при $\theta_{\mathrm{c}}<\theta \leqslant \hat{\theta}($ а), при $\theta>\check{\theta}$ (б) и график функции $3\left(\lambda_{3}^{\prime}\right)^{2}-1$ при $\hat{\theta}<\theta<\check{\theta}$ (в).

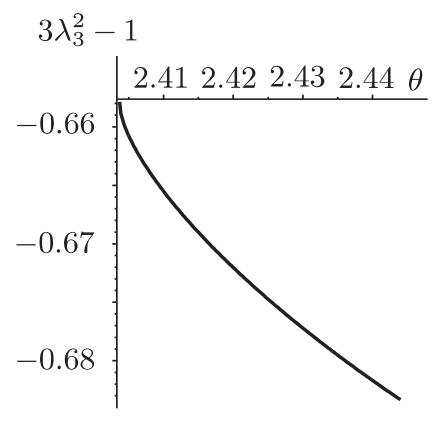

a

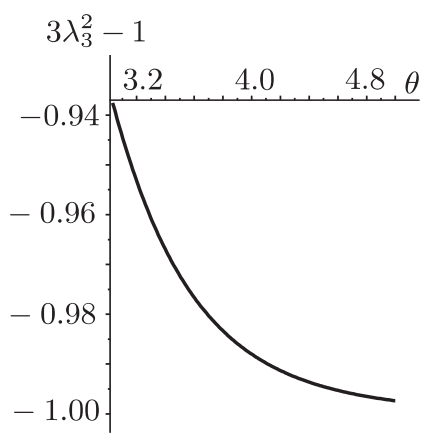

б

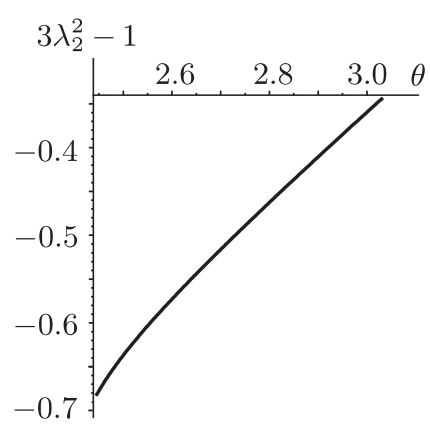

B

Рис. 4. График функции $3 \lambda_{3}^{2}-1$ при $\theta_{\text {c }}<\theta<2.444$ (а), при $\theta \geqslant \hat{\theta}$ (б) и график функции $3 \lambda_{2}^{2}-1$ при $2.444<\theta<\hat{\theta}$ (в). 


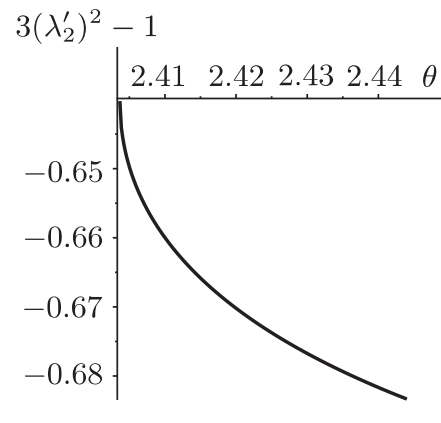

a

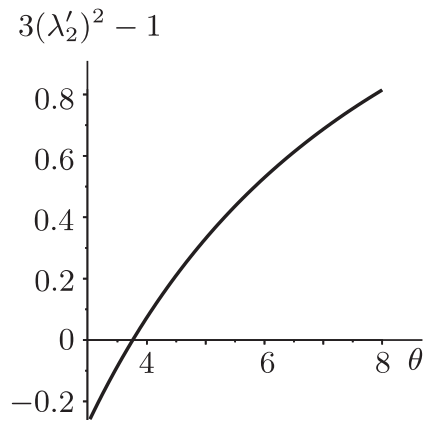

б

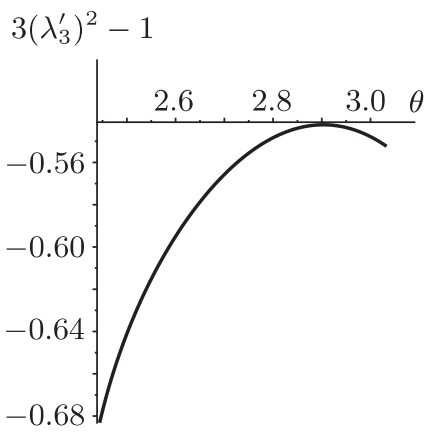

B

Рис. 5. График функции $3\left(\lambda_{2}^{\prime}\right)^{2}-1$ при $\theta_{\mathrm{c}}<\theta<2.444$ (а), при $\theta \geqslant \hat{\theta}$ (б) и график функции $3\left(\lambda_{3}^{\prime}\right)^{2}-1$ при $2.444<\theta<\hat{\theta}$ (в).

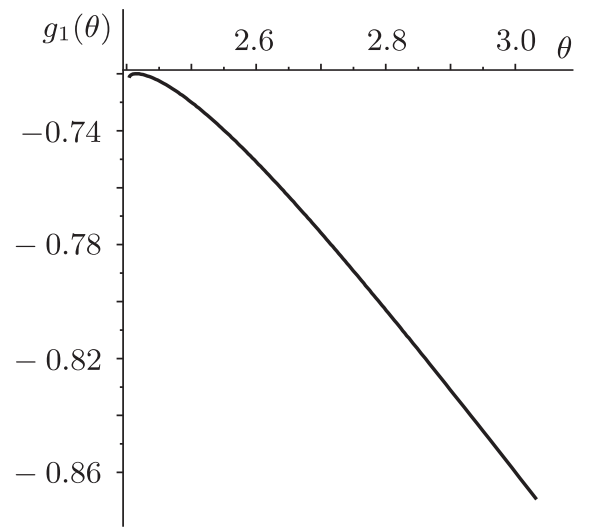

a

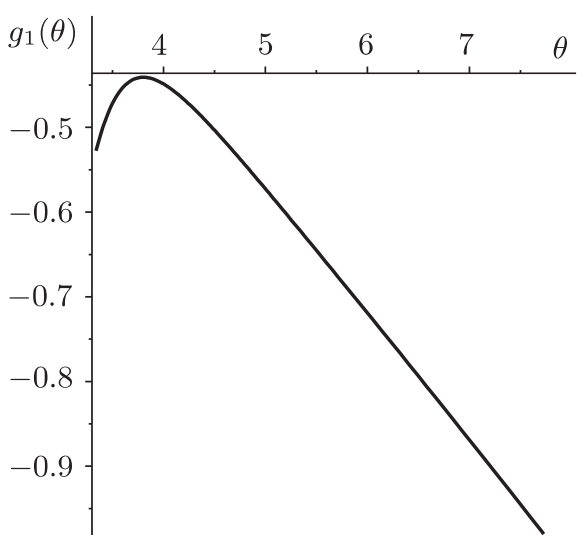

б

Рис. 6. График функции $g_{1}(\theta)$ при $\theta_{\text {c }}<\theta \leqslant \hat{\theta}$ (а) и при $\check{\theta}<\theta \leqslant \theta^{(3)}$ (б).

5.2. Условия крайности мер. Проверим условие $3 \kappa \gamma<1$, при котором мера $\mu_{0}$ является крайней. В этом случае $\kappa=\frac{\theta-1}{\theta+2}, \gamma=\frac{\theta-1}{\theta+1}$. Отсюда мера $\mu_{0}$ является крайней при $\theta \in\left(\frac{9-\sqrt{73}}{4}, \frac{9+\sqrt{73}}{4}\right)$.

Проверим крайность меры $\mu_{1}$. Пусть $z_{2}(\theta)>1$. Тогда из графиков можно увидеть, что $\kappa=\max \{b, c\}=c$ при $\theta_{\text {с }}<\theta \leqslant \hat{\theta}, \check{\theta}<\theta<\theta^{(3)} \approx 7.7305, \theta>\theta^{(4)} \approx 7.895$ и $\kappa=\max \{b, c\}=b$ при $\theta^{(3)}<\theta<\theta^{(4)}$. Кроме того, при $z_{2}(\theta)>1$ имеем $\gamma=\frac{\theta-1}{\theta+1}$ и для условия крайности при $\kappa=c$ получаем неравенство

$$
g_{1}(\theta)=3 \kappa \gamma-1=3 \cdot \frac{z_{2}\left|\theta-\sqrt[3]{z_{2}}\right|+\left|1-\theta \sqrt[3]{z_{2}}\right|+\left|1-\sqrt[3]{z_{2}}\right|}{2\left(\theta z_{2}+2\right)} \cdot \frac{\theta-1}{\theta+1}-1<0,
$$

справедливое при всех $\theta_{\text {c }}<\theta \leqslant \hat{\theta}, \check{\theta}<\theta<\theta^{(3)}$ (см. рис. 6) и $\theta^{(4)}<\theta<\theta^{(5)} \approx 14.14214$ (см. рис. 7б). При $\kappa=b$ имеем неравенство

$$
g_{2}(\theta)=3 \kappa \gamma-1=3 \cdot \frac{(\theta-1) \sqrt[3]{z_{2}}}{\theta z_{2}+2} \cdot \frac{\theta-1}{\theta+1}-1<0,
$$

которое верно при любых $\hat{\theta}<\theta<\theta^{(4)}$ (см. рис. 7а). 


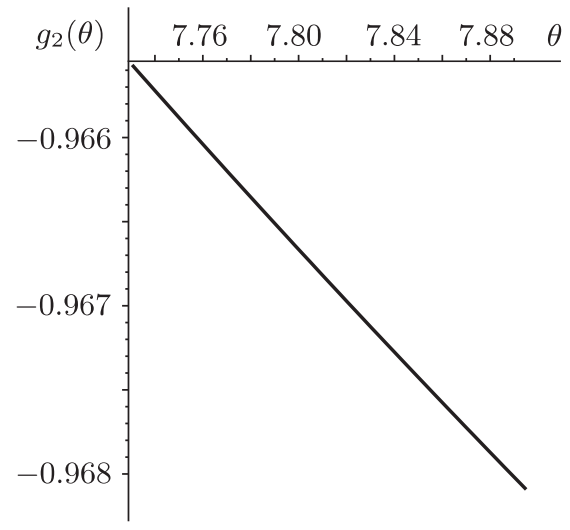

a

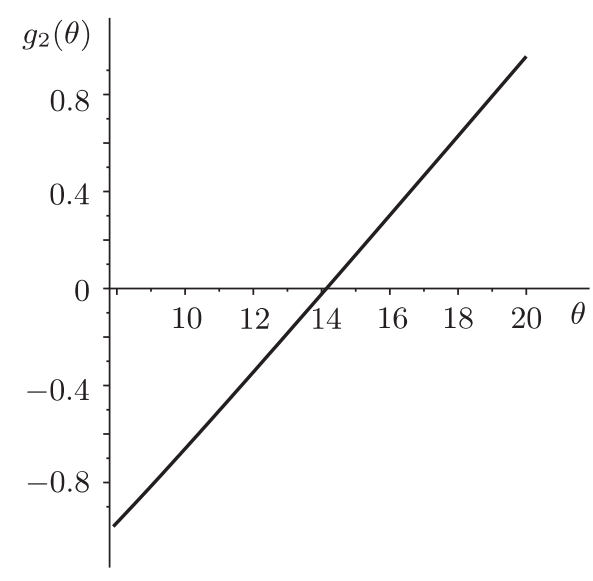

б

Рис. 7. График функции $g_{2}(\theta)$ при $\theta^{(3)}<\theta \leqslant \theta^{(4)}$ (а) и график функции $g_{1}(\theta)$ при $\theta>\theta^{(4)}($ б).

При $z_{2}(\theta)<1$ можно увидеть из графика, что $\kappa=\max \{b, c\}=b$, и для условия крайности мы получаем неравенство $g_{2}(\theta)<0$, которое верно, в частности, при любых $\hat{\theta}<\theta<\check{\theta}$ (см. рис. 7а). Таким образом, мера $\mu_{1}$ является крайней при любых $\theta_{\mathrm{c}}<\theta<\theta^{(5)}$.

Проверим крайность меры $\mu_{2}$. Пусть $z_{3}(\theta)>1$. Аналогично предыдущему случаю можно увидеть из графика, что $\kappa=c$ при $\theta_{\mathrm{c}}<\theta<\theta^{(1)}, \hat{\theta}<\theta<\theta^{(6)} \approx 3.74164$, $\theta>\theta^{(7)} \approx 4.10343$ и $\kappa=b$ при $\theta^{(6)}<\theta<\theta^{(7)}$. Кроме того, $\gamma=\frac{\theta-1}{\theta+1}$. Из условия крайности при $\kappa=c$ имеем

$$
g_{4}(\theta)=3 \kappa \gamma-1=3 \frac{z_{3}\left|\theta-\sqrt[3]{z_{3}}\right|+\left|1-\theta \sqrt[3]{z_{3}}\right|+\left|1-\sqrt[3]{z_{3}}\right|}{2\left(\theta z_{3}+2\right)} \cdot \frac{\theta-1}{\theta+1}-1<0,
$$

а при $z_{3}<1\left(\right.$ при $\left.\theta^{(1)}<\theta<\hat{\theta}\right)$ мы получаем $\kappa=b$ и условие крайности

$$
g_{5}(\theta)=3 \kappa \gamma-1=3 \cdot \frac{(\theta-1) \sqrt[3]{z_{3}}}{\left(\theta z_{3}+2\right)} \cdot \frac{\theta-1}{\theta+1}-1<0 .
$$

Анализируя графики функций $g_{4}(\theta)$ и $g_{5}(\theta)$, убеждаемся, что мера $\mu_{2}$ является крайней при $\theta \in\left(\theta_{\mathrm{c}}, \theta^{(8)}\right)$, где $\theta^{(8)} \approx 10.794118$ (см. рис. 8 и рис. 9$)$.

Проверим крайность меры $\mu_{3}$. Пусть $z_{2}(\theta)>1$. Тогда можно увидеть из графиков, что $\kappa=\max \left\{a^{\prime}, b^{\prime}, c^{\prime}\right\}=a^{\prime}$ при $\theta_{\text {с }}<\theta \leqslant \hat{\theta}$ и $\check{\theta}<\theta<\theta^{(9)} \approx 11.492$; $\kappa=\max \left\{a^{\prime}, b^{\prime}, c^{\prime}\right\}=c^{\prime}$ при $\theta>\theta^{(9)}$. Если $z_{2}(\theta)<1$, то $\kappa=\max \left\{a^{\prime}, b^{\prime}, c^{\prime}\right\}=b^{\prime}$ при $\hat{\theta}<\theta<\check{\theta}$. Таким образом, при $z_{2}(\theta)>1$ для условия крайности при $\kappa=a^{\prime}$ имеем неравенство

$$
g_{6}(\theta)=3 \kappa \gamma-1=3 \cdot \frac{(\theta-1)^{2} z_{2}}{\left((\theta+1) z_{2}+1\right)(\theta+1)}-1<0,
$$

которое верно при любых $\theta_{\text {c }}<\theta \leqslant \hat{\theta}, \check{\theta}<\theta<\theta^{(10)} \approx 3.8923$ (см. рис. 10 ). 


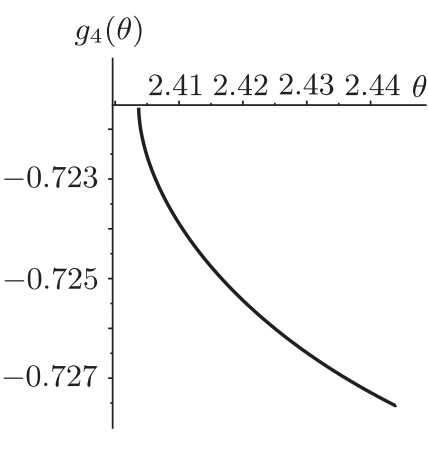

a

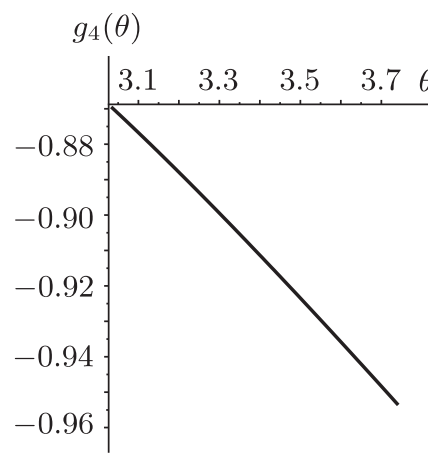

б

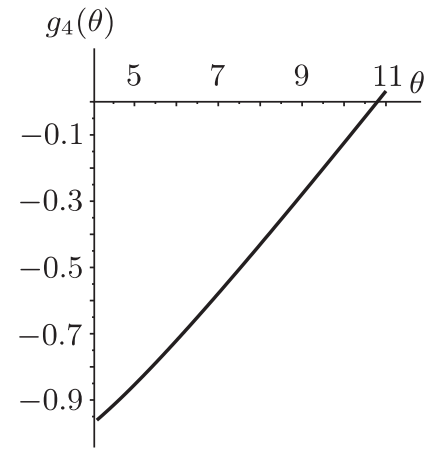

B

Рис. 8. График функции $g_{4}(\theta)$ при $\theta_{\mathrm{c}}<\theta \leqslant \theta^{(1)}$ (а), при $\hat{\theta}<\theta \leqslant \theta^{(6)}$ (б) и при $\theta>\theta^{(7)}($ б).

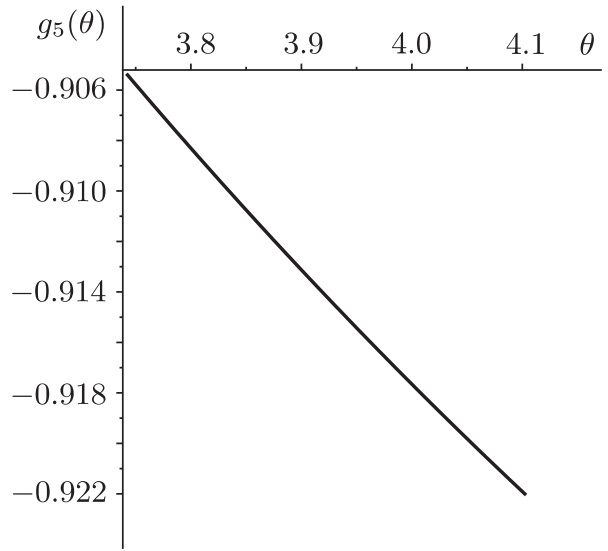

a

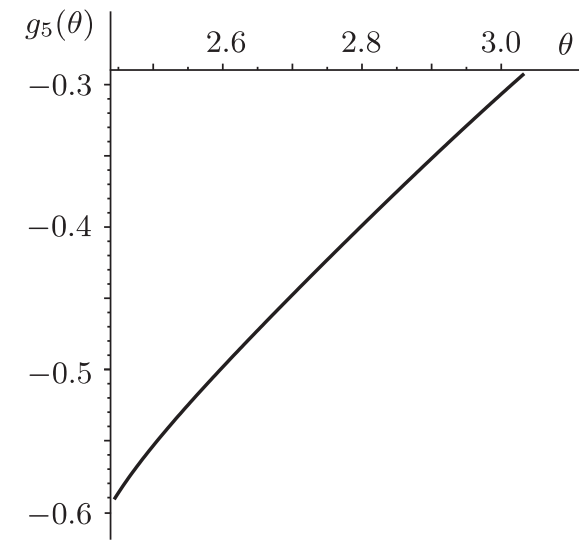

б

Рис. 9. График функции $g_{5}(\theta)$ при $\theta^{(6)}<\theta \leqslant \theta^{(7)}$ (а) и при $\theta^{(1)}<\theta<\hat{\theta}$ (б).

Если $\kappa=c^{\prime}$, то для условия крайности получаем неравенство

$$
g_{7}(\theta)=3 \kappa \gamma-1=3 \cdot \frac{z_{2} \cdot\left|\theta-\sqrt[3]{z_{2}}\right|+\left|1-\theta \sqrt[3]{z_{2}}\right|+z_{2}\left|1-\sqrt[3]{z_{2}}\right|}{2\left((\theta+1) z_{2}+1\right)} \cdot \frac{\theta-1}{\theta+1}-1<0,
$$

которое не имеет решений при любых $\theta>\theta^{(9)}$ (см. рис. 11a).

При $z_{2}(\theta)<1$ имеем $\kappa=b^{\prime}$ и для условия крайности получаем неравенство

$$
g_{8}(\theta)=\frac{3(\theta-1) \sqrt[3]{z_{2}}}{(\theta+1) z_{2}+1} \cdot \frac{\theta-1}{\theta+1}-1<0,
$$

которое верно при любых $\hat{\theta}<\theta<\check{\theta}$ (см. рис. 11б). Таким образом, мера $\mu_{3}$ является крайней при любых $\theta_{\text {с }}<\theta<\theta^{(10)}$.

Аналогично мерам $\mu_{1}, \mu_{2}$ и $\mu_{3}$ можно получить интервалы, где мера $\mu_{4}$ является крайней. Итак, справедлива следующая теорема. 


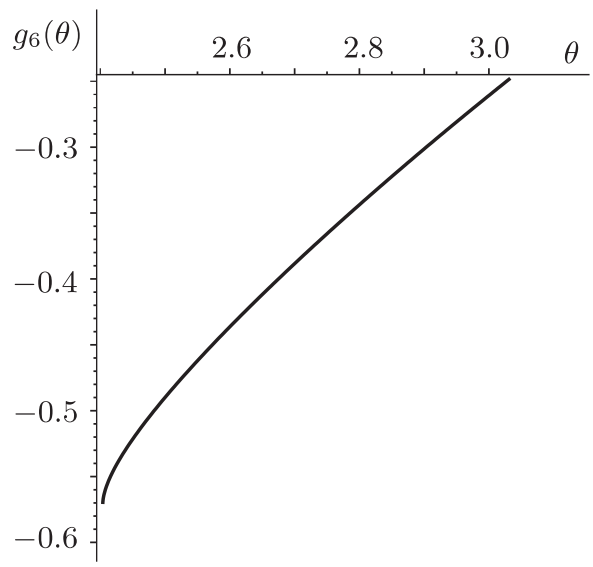

a

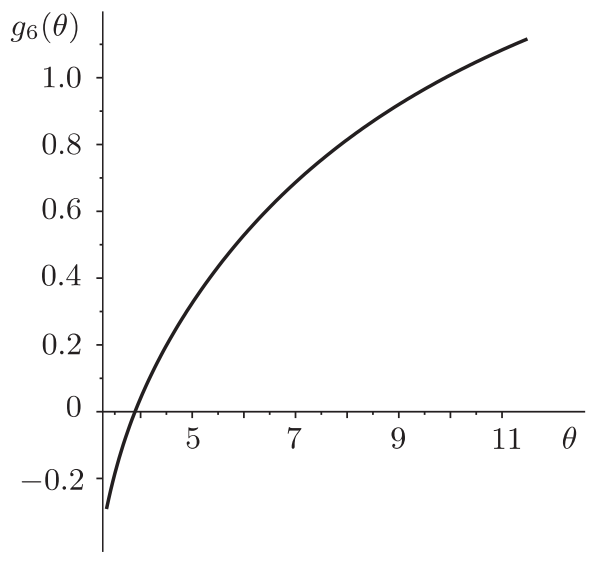

б

Рис. 10. График функции $g_{6}(\theta)$ при $\theta_{\mathrm{c}}<\theta \leqslant \hat{\theta}$ (а) и при $\check{\theta}<\theta<\theta^{(9)}$ (б).

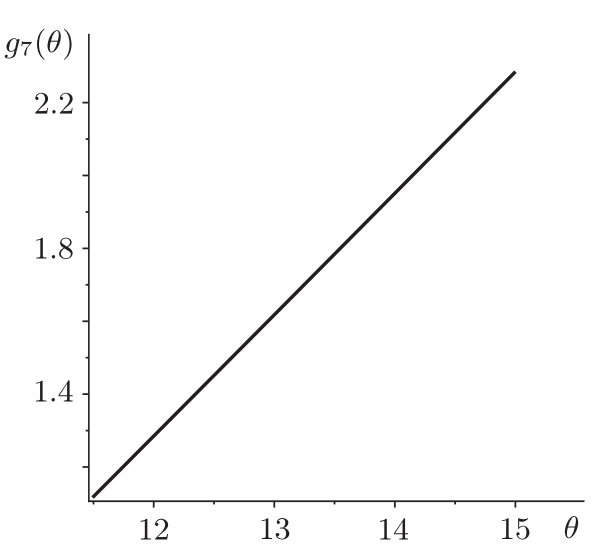

a

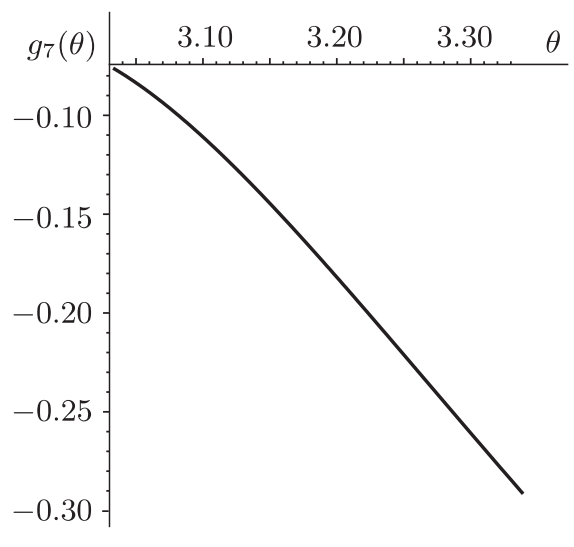

6

Рис. 11. График функции $g_{7}(\theta)$ при $\theta>\theta^{(9)}$ (а) и график функции $g_{8}(\theta)$ при $\hat{\theta}<\theta<\check{\theta}$ (б).

ТЕОрема 5. Пусть $k=3, q=3$ и J>0. Тогда верны следующие утверждения.

1. Мера $\mu_{0}$ является крайней при $0.114 \approx \frac{9-\sqrt{73}}{4}<\theta<\frac{4}{9+\sqrt{73}} \approx 4.386$ ине является крайней при $\theta>\frac{1}{2}(5+3 \sqrt{3}) \approx 5.0981$.

2. Меры $\mu_{1}$ и $\mu_{2}$ являются крайними при $\theta_{\mathrm{c}}<\theta<\theta^{(5)}$ u $\theta_{\mathrm{c}}<\theta<\theta^{(8)}$ coответственно.

3. Меры $\mu_{3}$ и $\mu_{4}$ не являются крайними при $\theta>\theta^{(0)} u \theta>\theta^{(2)}$ соответственно.

4. Мера $\mu_{3}$ является крайней при $\theta_{\mathrm{c}}<\theta<\theta^{(10)}$.

5. Мера $\mu_{4}$ является крайней при $\theta_{\mathrm{c}}<\theta<\theta^{(11)}$, где $\theta^{(11)} \approx 3.74608$. 
ЗАмЕЧАНИЕ 3. Отметим, что остаются следующие зазоры, т. е. интервалы, где неизвестно, являются ли меры крайними или нет:

для меры $\mu_{0}$ это интервалы $\left(0, \frac{9-\sqrt{73}}{4}\right) \approx(0,0.114)$ и $\left(\frac{9+\sqrt{73}}{4}, \frac{5+3 \sqrt{3}}{2}\right) \approx(4.386,5.0981)$;

для меры $\mu_{1}$ это интервал $\theta>\theta^{(5)} \approx 14.14214$;

для меры $\mu_{2}$ это интервал $\theta>\theta^{(8)} \approx 10.794118$;

для меры $\mu_{3}$ это интервал $\left(\theta^{(10)}, \theta^{(0)}\right) \approx(3.8923,3.994)$;

для меры $\mu_{4}$ это интервал $\left(\theta^{(11)}, \theta^{(2)}\right) \approx(3.74608,3.759659)$.

Благодарности. Авторы выражают свою признательность рецензенту за полезные замечания.

\section{Список литературы}

[1] Х.-О. Георги, Гиббсовские меры и фазовые переходь, Мир, М., 1992.

[2] К. Престон, Гиббсовские состояния на счетных множествах, Мир, М., 1977.

[3] Я.Г. Синай, Теория фазовых переходов. Строгие результаты, Наука, М., 1980.

[4] П. М. Блехер, Н. Н. Ганиходжаев, "О чистых фазах модели Изинга на решетках Бете", Теория вероятн. и ее примен., 35:2 (1990), 220-230.

[5] P. M. Bleher, J. Ruiz, V.A. Zagrebnov, "On the purity of the limiting Gibbs state for the Ising model on the Bethe lattice", J. Statist. Phys., 79:1-2 (1995), 473-482.

[6] Н. Н. Ганиходжаев, "О чистых фазах ферромагнитной модели Поттса с тремя состояниями на решетке Бете второго порядка", ТМФ, 85:2 (1990), 163-175.

[7] Н. Н. Ганиходжаев, "О чистых фазах ферромагнитной модели Поттса на решетке Бете", Докл. АН РУз, 6-7 (1992), 4-7.

[8] U. A. Rozikov, Gibbs Measures on Cayley Trees, World Sci., Singapore, 2013.

[9] N. N. Ganikhodjaev, U. A. Rozikov, "The Potts model with countable set of spin values on a Cayley tree", Lett. Math. Phys., 75:2 (2006), 99-109.

[10] У. А. Розиков, Р. М. Хакимов, "Периодические меры Гиббса для модели Поттса на дереве Кэли", ТМФ, 175:2 (2013), 300-312.

[11] Р. М. Хакимов, "О существовании периодических мер Гиббса для модели Поттса на дереве Кэли", Узбек. матем. журн., 2014, № 3, 134-142.

[12] R. M. Khakimov, "New periodic Gibbs measures for $q$-state Potts model on a Cayley tree [Новые периодические меры Гиббса для модели Поттса с $q$-состояниями на дереве Кэли]", Журн. СФУ. Сер. Матем. и физ., 7:3 (2014), 297-304.

[13] C. Külske, U. A. Rozikov, R. M. Khakimov, "Description of all translation-invariant splitting Gibbs measures for the Potts model on a Cayley tree", J. Statist. Phys., 156:1 (2014), 189-200, arXiv: 1310.6220.

[14] C. Külske, U.A. Rozikov, "Fuzzy transformations and extremality of Gibbs measures for the Potts model on a Cayley tree", Random Structures and Algorithms, 50:4 (2017), 636-678, arXiv: 1403.5775.

[15] Р. М. Хакимов, Ф. Х. Хайдаров, “Трансляционно-инвариантные и периодические меры Гиббса для модели Поттса на дереве Кэли", ТМФ, 189:2 (2016), 286-295.

[16] C. Külske, M. Formentin, "A symmetric entropy bound on the non-reconstruction regime of Markov chains on Galton-Watson trees", Electron. Commun. Probab., 14, paper no. 57 (2009), 587-596.

[17] H. Kesten, B. P. Stigum, "Additional limit theorem for indecomposable multi-dimensional Galton-Watson processes", Ann. Math. Statist., 37:6 (1966), 1463-1481. 
[18] E. Mossel, "Survey: information flow on trees", Graphs, Morphisms and Statistical Physics (Piscataway, NJ, March 19-21, 2001), DIMACS Series in Discrete Mathematics and Theoretical Computer Science, 63, eds. J. Nešetril, P. Winkler, AMS, Providence, RI, 2004, $155-170$.

[19] F. Martinelli, A. Sinclair, D. Weitz, "Fast mixing for independent sets, coloring, and other models on trees", Random Structures and Algoritms, 31:2 (2007), 134-172.

Поступила в редакцию 17.08.2017, после доработки 11.10.2017 